Das Zinkperhydrol läBt sich also mit Hilfe von gesăuerter Jodkaliumlösung innerhalb einer Reaktionsdauer von 10-15 Minuten bestimmen.

Die Berechnung der Resultate entspricht den Gleichungen:

$$
\begin{aligned}
& \mathrm{Zn} \mathrm{O}_{\mathrm{g}}+2 \mathrm{HJ}=2 \mathrm{~J}+\mathrm{Zn}\left(\mathrm{OH}_{\mathrm{g}}\right. \\
& 1 \mathrm{ZnO}_{2} \quad n=2 \mathrm{~J} \\
& 97 \mathrm{~g} n=2 \mathrm{~J} \\
& 48,5 n \quad n=1 \mathrm{~J} \\
& 4,85 n \quad n=1 / 10 \mathrm{~J}=1000 \mathrm{ccm} n / 10 \mathrm{~J} \\
& 0,00485 \pi, \quad \pi=1 \mathrm{ccm} \mathrm{J} / 10 \\
& \text { also } 20,07 \mathrm{ccm} \mathrm{z} / 10 \mathrm{~J}=0,0971 \mathrm{~g} \mathrm{Zn} \mathrm{O} \text {. }
\end{aligned}
$$

Das angewandte Präparat war demnach $48,55 \%$ ig.

Schließlich mag noch angefugt werden, daß Natrinmsuperoxydpu äparate wegen der stirmischen Sauerstoffentwickelung beim Zusammenbringen mit Wasser durch Jodlauge nicht bestimmbar sind.

Indifferent gegen letztere verhalten sich die Persulfate, analog deren Verhalten gegentiher Permanganat.

\title{
Ueber Reinigung von Wasser mittels Eisenhydroxyd und ein einfaches und billiges Verfahren zur Herstellung einer hierzu geeigneten Lösung von kolloidalem Eisenhydroxyd ohne Dialyse.
}

Von A potheker H. Schweikert - Bonn.

(Eingegangen den 19. XI. 19C6.)

Einem einfachen und billigen Verfahren zur Reinigung von Wasser kommt unzweifelhaft für die Wasserversorgung besonders von Städten und Ortschaften eine große Bedeutung zu.

Ich glaube nun, daß bis jetzt kaum ein anderes Mittel zu diesem Zwecke bekannt ist, welches mit Einfachheit, verhältnismäßiger Billigkeit und völliger Gefahrlosigkeit gunstigere Wirkung verbindet als das Eisenhydroxyd.

Schon längst hat man zur Reinigung von Wässern die Erzeugung von Niederschlägen in denselben durch Zusatz von Chemikalien angewandt, um durch diese Niederschläge die in dem Wasser vorhandenen Schwebestoffe niederzureißen no so das Wasser zu klären. Aber diese Reinigung und Klärung war meistens nur eine mechanische, um an und für sich brauchbare Wässer lediglich von den mehr oder minder 
zufällig vorhandenen festen Partikelchen (Lebm, Ton, Fett etc.) zu befreien ${ }^{\mathrm{t}}$ ).

$\mathrm{Zu}$ diesem Zwecke benutzt man die verschiedensten Stoffe. So führt Koenig ${ }^{2}$ ) nicht weniger als 75 derartige Verfabren an, daranter uicht weniger als 30 , welche sich der Eisenverbindungen entweder für sich allein oder in Kombination mit anderen Stoffen bedienen.

Der Wirkung dieser chemischen Fällungsmittel steht Koenig im allgemeinen ziemlich skeptisch gegentber. Er sagt dartiber: „Durch die meisten derselben wird ein fauliges Abwasser zwar mehr oder weniger geruchlos, aber auf die gelösten Stoffe sind alle Fallungsmittel mehr oder weniger obne Einfluß; unter Umständen werden sogar, besonders bei Anwendung von überschlissigem Kalk, die Schwebestoffe zam Teil in Lösung abergeführt" ${ }^{8}$ ).

Wohl weist Koenig aber darauf hin), daß die Bildang der Niederschläge von Eisenoxydoxydulhydrat oder von Eisenoxydhydrat in dem zu reinigenden Wasser die Ausfällung der gelösten organischen Stoffe begünstigt.

Die zur Reinigung von Trinkwasser brauchbaren und angewandten Methoden werden von diesem Autor ${ }^{5}$ ) in $3 \mathrm{Abteilungen} \mathrm{gebracht.} \mathrm{In}$ der ersten finden si h solche, welche bloß eine chemische Wirkung âußern, ohne die Bestandteile des Wassers als solche wesentlich zn verändern. Hierzu gehört in erster Linie das Eisenchlorid mit oder ohne Anwendung von Kalk oder Natriumbikarbonat; diese Methode interessiert uns hier um deswillen besonders, weil der dabei entstehende Niederschlag von Eisenhydroxyd mit dem Niederschlag durch die von mir empfohlene Eisenhydroxydlösung übereinstimmt.

Nach Kirchner ${ }^{6}$ ) wurde vom Preußischen Kriegsministerium bereits im Jahre 1878 ein Zusatz von $0,45-0,675 \mathrm{~g}$ Eisenchlorid und $0,20-0,30 \mathrm{~g}$ Natriumbikarbonat für 1 Liter Wasser vorgeschlagen. Plagge ${ }^{7}$ ) findet dieses Verfabren als außerordentlich wirksam und zur Zeit seiner Einführung von den Truppen nur nicht gentigend gewtirdigt. Der sich bildende voluminöse Niederschlag von Eisenoxydhydrat reißt nach Plagge nicht nur die gröberen Trübungen des Wassers, sondern

1) Hans Reisert, Köln a. Rh., Abt. III, Wasserreinigungsanlagen.

2) Koenig, Die Verunreinigung der Gewässer etc., 2. Anfl. 1899. Band I, S. $35 \overline{3} \mathrm{ff}$.

-) Koenig, Die Veranreini jung des Gawasser etc., Band I, S. 360.

4) Ebenda, S. 361 .

8) Ebenda, S. $191 \mathrm{ff}$.

0) Kirchner, GrundriB d. Mulit. G ssundheitspflege 1891, S. 162.

7) Plagge in Veröffentl. a. d. Geb. d. Milit.-Sanitatsw., herauggegeben von der Mediz. Abt. d. Kőnigl. Kriegsministeriams, Heft 9. 
auch nahezu sämtliche Bakterien mit zu Boden, und die obenstehende klare Flüssigkeit ist fast vollkommen keimfrei.

Koenig ${ }^{\text {) }}$ aber kommt zum Schluß seiner Frörterungen über die Reinigung des Trinkwassers dorch Chemikalien za folgendem Urteil: "Im übrigen gilt, wenn schon die Anwendung von Chemikalien zur Reinigung von Schmutzwasser als ein Notbehelf angesehen werden muß, dieses besonders fur Reinigung von Trinkwasser. Die Wirkung ist eine unsichere, und sind die Verfahren in der praktischen Ausführung um deswillen schwierige, weil die Menge der Zusătze jedesmal genau dem zu reinigenden Wasser angepaßt werden muß. Setzt man zu wenig Chemikalien zu, so bleibt die reinigende oder sterilisierende Wirkung mehr oder weniger ganz aus, setzt man aber etwas zaviel zu, so wird das Wasser leicht ungeniebbar".

Auch die eben erwäbnte von Plagge als so außerordentlich wirksam befundene Methode mit Eisenchlorid und Natrinmbikarbonat leidet an diesem letzten von Koenig gerügten Mangel. Die beiden Ijösungen müssen genau abgewogen werden, weil sonst die Gefahr entsteht, daß entweder das Wasser eisenhaltig wird, wenn man za viel Eisenchlorid zusetzt, oder durch äberschüssiges Natriumbikarbonat alkalisch wird und laugenartigen Geschmack annimmt. Ferner wird durch das zugefügte Natriumbikarbonat eine entsprechende größere Menge Chlornatrium gebildet, welche im Wasser gelöst bleibt und dieses stark chlorhaltig macht. Drittens aber entwickelt sich durch das zugefügte Natriumbikarbonat bei der Zersetzung des Eisenchlorids eine entsprechende Menge von Kohlensäure, welche sich in feinen Bläschen an den gebildeten Eisenhydroxyd-Niederschlag ansetzt und bewirkt, daß sich derselve nur schwierig zu Boden setzt, denselben vielmehr in der Schwebe erhält oder wohl gar an die Oberflache des Wassers hebt, und nur durch lăngeres kräftiges Umrühren kann dieser Uebelstand in etwa beseitigt werden.

Alle diese, wie au؛h die anderen von Koenig gerügten Mängel bei Anwendung von Chemikalien treten jedoch bei Anwendung der kolloidalen Eisenbydroxydlösung garnicht oder doch nur in ganz verschwindendem Maße auf.

Die kolloidale Eisenhydroxydlösung hat die hervorragende Eigenschaft sowohl darch sehr geringe Mengen kaustischer oder kohlensaurer Alkalien und alkalischer Erden, wie auch durch sebr geringe Mengen von Mineralsäuren und durch die meisten Neutralsalze koaguliert und vollständig gefällt zu werden. $\mathrm{Da}$ aber die zur Wasserversorgung benutzten Wässer nie ganz frei von solchen Salzen sind, so wird das

1) Koenig, Die Verunreinigung der Gewässer etc., Band I, S. 194. 
Eisenhydroxyd aus seiner Lösung dadurch vollständig ausgeschieden, und liegt eine Gefahr der Verunreinigung des Wassers durch Eisen hierbei nicht vor, wenn man nicht unvernünftig viel von der Eisen. hydroxydlösung zusetzt. Selbst wenn man das Mebrfache der im all. gemeinen zur Reinigung des Wassers ausreichenden Menge $(1: 1000)$ zusetzt, wird in der Regel alles Eisenhydroxyd gefällt, und das Wasser ist nach dem Absetzen oder Abfiltrieren vollkommen frei von Eisenoxyd.

Andererseits aber geht das Eisenhydroxyd mit den meisten im Wasser vorkommenden gelösten organischen Substanzen, besonders auch mit Eiweiß-und anderen Protein-Substanzen, Huminstoffen usw. unlösliche Verbindungen ein, so daß sie, wenn sie im Wasser vorhanden sind, mit niedergeschlagen werden, das Wasser also dadurch davon befreit wird.

Ein weiterer Vorzug der kolloidalen Eisenhydroxydlösung aber ist, daß das Wasser, wenn es etwa Sulfate, z. B. Gips in größerer Menge enthält, mebr oder weniger durch dieselbe davon befreit wird, indem sie sich zum 'Teil mit den Sulfaten umsetzt und mit niederfallendes basisch-schwefelsanres Eisenoxyd bildet.

$\mathrm{Da}$ aber weiter die kolloidale Eisenhydroxydlösung nur einen sehr geringen Gehalt an Chlor hat, nämlich bei einem Gehalte von $3,5 \%$ Eisen nur höchstens $0,6-0,7 \%$ Chlor, und der niedertallende Niederschlag von Eisenhydroxyd außerdem noch ziemlich stark chlor. haltig ist, so ist auch die Vermehrung der Chlorverbindungen im Wasser verschwindend klein.

Diese hervorragenden Eigenschaften der kolloidalen Eisenhydroxdlösung zur Reinigung von Wasser sind schon früher mehrfach erkannt worden').

Auch aus den von mir angestellten und von Herrn Dr. Gronover") bestätigten chemischen Untersuchungen von Elbwasser, welches ich dem freundlichen Entgegenkommen des Magistrats der Stadt Magdeburg in größerer Menge verdankte, ergibt sich, daß der Gebalt an sogen. organischer Substanz nach Zusatz von Eisenhydroxydlösung im Verhältnis von 1:1000 auf weniger als die Hälfte berabging, bei einem $Z$ usatz von 2:1000 nnd 3:1000 aber auf ca. den vierten Teil, und daB dabei der Chlorgehalt nur um 1 Teil anf 100000 Teile Wasser zunahm. Die Tabelle S. 16 gibt tuber das Resultat der chemischen Untersuchung näheren Aufschluß.

1) Hager, Handbuch der pharm. Praxis, Ergänzungaband, 1883, S. 103, sowie Biltz u. Krobnke, Berichte d. Chem. Ges. 1904, Heft 7, S. 1751, III.

2) z. Z. Direktor d. Städt. Untersuchungsamtes in Müblhausen i. E. 
Gleich günstig war das Ergebnis der bakteriologischen Untersuchungen des Elbwassers im hygienischen Institut der Königlichen Universität zu Bonn ${ }^{1}$ ).

\begin{tabular}{c||c|c|c|c|c|c|}
\hline \hline $\begin{array}{c}\text { In 1 ccm Wasser sind } \\
\text { gefunden worden: }\end{array}$ & $\begin{array}{c}\text { Roh- } \\
\text { wasser }\end{array}$ & \multicolumn{3}{|c|}{$\begin{array}{c}\text { Mit Eisenhydroxyd geklärtes } \\
\text { Wasser im Verhältnis von }\end{array}$} \\
\cline { 2 - 7 } & $0,5: 1000$ & $1: 1000$ & $2: 1000$ & $3: 1000$ \\
\hline Bakterienkeime $\ldots \ldots \ldots \ldots$ & 1470 & 750 & 364 & 178 & 72
\end{tabular}

Auch hier ist die Zahl der Keime in dem 1:1000 geklärten Wasser aut ca. 1/4; bei dem 2:1000 geklärten auf ca. 1/8, und bei dem 3: 1000 geklärten sogar auf 1/20 herabgemindert worden.

\begin{tabular}{|c|c|c|c|c|c|c|}
\hline \multirow{3}{*}{$\begin{array}{l}\text { In } 100000 \text { Teilen } \\
\text { Elbwasser sind } \\
\text { gefunden worden: }\end{array}$} & \multicolumn{2}{|c|}{$\begin{array}{c}\text { I. } \\
\text { Roh a asser }\end{array}$} & \multicolumn{4}{|c|}{$\begin{array}{l}\text { II. Mit Eisenhydroxyd geklärt } \\
\text { im Verhältnis von }\end{array}$} \\
\hline & \multirow{2}{*}{ Sehweikert } & \multirow{2}{*}{ Gronorer } & \multicolumn{2}{|c|}{$1: 1000$} & \multirow{2}{*}{$\frac{2: 1000}{\text { Schweikert }}$} & \multirow{2}{*}{\begin{tabular}{|l|}
$3: 1000$ \\
Schweiker
\end{tabular}} \\
\hline & & & Schweilert & Gronorer & & \\
\hline Abdampfrückstand & 52,0 & 54,4 & 49,7 & 54,7 & 50,7 & 51,8 \\
\hline Glühverlust . & 8,5 & 9,8 & 10,0 & 10,7 & 6,2 & 8,1 \\
\hline Organische Substanz ${ }^{2}$ ) & 14,0 & 11,0 & 6,0 & 4,8 & 4,25 & 3,75 \\
\hline Chlor . . . . . . . & 17,4 & 17,2 & 18,4 & 18,25 & 18,8 & 19,9 \\
\hline Schæefelsäure & 5,7 & 4,9 & 5,6 & 4,6 & 5,5 & 5,6 \\
\hline Salpotersäure . & $?$ & 0,24 & $?$ & 0,24 & $?$ & $?$ \\
\hline Ammoniak . . & fehlt & fellt & feblt & feblt & fehlt & fehlt \\
\hline Salpetrige Säure . & & $\pi$ & $n$ & $n$ & $\eta$ & $n$ \\
\hline $\left.\operatorname{Eisen}^{8}\right) \ldots \ldots$ & Spur & Spur & Spur & Spur & Spur & Spur \\
\hline
\end{tabular}

Weiter hat Herr Dr. Hugo Fischer ? mit dem Elbwasser and der ihm von mir übergebenen Eisenhydroxydlösung bakteriologische Untersuchungen angestellt. Er berichtet $u$. a.: "In den nicht (mit Eisenhydroxydlösung) behandelten Kulturen waren stets eine Menge Keime, pro Tropfen mehrere Hunderte in Durchschnitt, nachweislich;

1) Ausgeführt durch Herrn Privatdozent Dr. Selter.

2) Die Bestimmung der organischen Substanz erfolgte mit Kaliumpermanganat, und zwar von Dr. Gronover in alkalischer Lösung, während ich selbst nach Kubel in saurer Lösung bestimmte. Dadurch dürfte sich die Differenz in beiden Bestimmungen erklüren. Der starke Coloridgehalt des Wassers wird, wie wohl anzunehmen ist, in saurer Lösung das Resultat ungünstig beeinflubt haben, und werden auch dementsprechend die bei dem im Verhältnis 2:1000 und 3:1000 geklärten Wasser gefundenen Zablen für organische Substanz herabzusetzen sein.

-) Unwägbar, nur im Abdampfrückstand nachweisbar.

4) Derzeit Privatdozent der Botanik u. d. Bakteriologie an der Königl. Universităt Bonn and an der Landw. Akademie Bonn-Poppelsdorf. 
im Filtrat war die Keimzahl mindestens aut etwa den tünften 'Tuil herabgesetzt, manche Schälchen erwiesen sich als völlig steril; in einem Versuch z. B. ging in drei Schälchen zusammen nur ein einziger Keim aut".

Als ganz besonders wichtig für die hygienische Seite der Frage erscheint eine Abhaudlung von $O$. Muller ${ }^{1}$ ): „Ueber den Nachweis von Typhusbazillen im Trinkwasser durch Fallung mit Eisenoxychloridlösung", aus welcher hervorgeht, daß eine Eisenoxychloridlösung (bezw. Eisenhydroxydlösung) eine sehr hohe Fällungskraft für Typhusbazillen besitzt. Muller hat 3 Liter reines Wasser künstlich mit einer bestimmten Anzahl Typhuskeime infiziert, dann mit $5 \mathrm{ccm}$ Eisenlösung gefällt und im Niederschlage fast die gesamte Menge der Typhusbazillen wiedergefunden, während das abfiltrierte Wasser davon frei gefunden warde.

Nach alledem dürfte die ausgezeichnete Wirksamkeit der kolloidalen Eisenhydroxydlösung für die Reinigung von Wasser außer allem Zweifel stehen.

Ich möchte noch mit einigen Worten auf die Reinignng des Wassers durch Sandfiltration, welche ja bisher die im großen am meisten angewandte Reinigungsmethode ist, eingehen.

Die Wirkung der Sandfiltration besteht nach C. Piefk ${ }^{2}$ ): erstens in der mechanischen, d. h. in der Zurückhaltung der Schwebestoffe; zweitens in der sogen. physiologischen, d. h. in der moglichst vollständigen Zurückhaltung der Mikroorganismen aller Art; von einer frischen Sandschicht werden diese nur unvollkommen zurückgehalten, vollkommen erst dann, wenn sich die sogen. "Schleimschicht" ${ }^{*}$ auf den Filtern gebildet hat; aber selbst diese Schmutzdecke, welche bei Filtration unreiner Wässer auf der Oberfäche des Filters entsteht, kann nicht verhindern, daß der unter ihr befindliche sterile Sand längere Zeit für Mikroorganismen durchlassig bleibt; drittens in der chemischen Wirkung, d. h. in der Umsetzung und Oxydation der gelösten, besonders der organischen Stoffe. Diese Wirkung ist aber nach $\mathrm{K}$ oe $\mathrm{n}$ ig's Angaben nur gering.

Aus den Ausführungen Koonig's geht uberhaupt hervor, daß die Sandfiltration nach allen drei angegebenen Richtungen hin unvollkommen wirkt. Dazu kommt, daß nach der Reinigung der Sandfilter das filtrierte Wasser so lange wieder einen hơheren Keimgehalt besitzt, bis sich die Schleimschicht von neuem gebildet hat ${ }^{8}$ ). Die Gewinuung eines keimfreien oder keimarmen Filtrats hängt also ganz

1) Zeitschr. f. Hygiene u. Infokt.-Krankh., Band 51 (1905), S. 1 ff.

2) Koenig, Die Verunreinigung der Gewåsser etc., Band I, S. 133.

8) E.benda, S. 135.

Arch. d. Pharm. CCXXXXV. Hds. 1. Heft. 
von der Dicke und Filtrierfähigkeit der vorbandenen Schleimschicht $\mathrm{ab}$ : wenn die Schleimschicht aber eine gewisse Stärke erreicht hat, so läßt sie kein Wasser mehr durch; sie muß dann entfernt, bezw. der Sand gereinigt werden.

C. $P$ ief $k \mathrm{e}^{1}$ ) hat auch Versuche darüber angestellt, welche von den drei Hautdecken, aus welchen sich die Schleimschicht bildet, namlich Algen, Lehm und Eisenoxyd, die Bakterien - er wählte den Bacillus violaceus - am meisten zuriuckhalten; er fand die Lebmdecke am besten wirkend, von 63165 Keimen im Rohwasser blieben im Filtrat nur 19 in $1 \mathrm{ccm}$. Aber auch die Eisenoxyddecke wirkte fast ebenso günstig; es blieben im Filtrat nur 25 in $1 \mathrm{ccm}$, whthrend bei der Algendecke immerhin 45 blieben.

Nach den Versuchen von C. Frănkel und C. Piefke ${ }^{2}$ ) sind die Sandfilter keine keimdicht wirkenden Apparate; weder die gewöhnlichen Wasserbakterien noch auch Typhusbazillen und Cholerabakterien werden von denselben mit Sicherheit zurückgehalten. Die Menge der in das Filtrat übergehenden Mikroorganismen ist abhängig von der Anzahl der im unfiltrierten Wasser vorhandenen Keime und von der Schnelligkeit der Filtration. Anfang und Ende einer jeden Gebrauchsperiode des Filter's sind besonders gefahrliche Zeiten; im ersteren Falle haben die Filter noch nicht ihre volle Leistungsfähigkeit erlangt, im letzteren Falle begünstigt die Pressung der oberen Filterschichten, vielleicht auch das selbständige Durchwachsen der Bakterien ein Abwärtssteigen der Mikroorganismen.

Es geht hieraus klar hervor, daß bei den Sandfiltern die "Schleimschicht" - nach den eben erwähnten Versuchen von C. P i efke - durch eine Eisenhydroxydschicht erfolgreich ersetzt werden kann.

Da aber weiter die Filtration um so wirksamer ist, je langsamer sie vor sich geht, so sind andererseits ausgedehntere Filteranlagen notwendig, um die nötige Menge Wasser zu liefern.

Ich habe nun, allerdings rur im kleinen und mit Papierfiltern, Versuche uber die Filtrationsgeschwindigkeit angestellt und zwar:

1. bei Rohwasser;

2. bei mit Eisenhydroxyd geklärtem Wasser, und hierbei je nachdem

a) der Eisenhydroxyd-Niederschlag mit auf das Filter gebracht wird, also gewissermaßen die ${ }_{n}$ Schleimschicht" ersetzt;

b) das Eisenhydroxyd sich abgesetzt hat und nur das geklärte Wasser auf das Filter gelangt.

1) Koenig, Die Verunreinigung der Gawässer etc., Band I, S. 136 bezw. Zeitschr. f. Hygiene u. Iofekt.-Krankh., Band 16 (1894), S. 181.

2) Zeitschr. f. Hygiene etc. 1890, Bd. 8, 1. 
Angewandt wurde, um ein möglichst leichtes Ablaufen des Filtrates zu erzielen, und die ganze Filterfläche wirken zu lassen, ein sorgtältig sternförmig gefaltetes Filter von gut durchlassendem doppeltstarkem Filtrierpapier von Schleicher \& Schüll No. 598. Der Flächeninhalt des Filters, welches während des Versuches immer möglichst voll gehalten wurde, betrug $500 \mathrm{qcm}$. Die Ergebnisse dieser Versuche ergeben sich aus der nachstehenden Tabelle:

\begin{tabular}{|c|c|c|c|c|c|c|c|c|c|c|c|c|}
\hline Es filtrierten: & 1 & 2 & 3 & 4 & 5 & 6 & 7 & 8 & 9 & 12 & 15 & Liter \\
\hline $\begin{array}{l}\text { 1. Rohwasser } \\
\text { in: }\end{array}$ & 2 & 4 & $61 / 2$ & $|101 / 2|$ & $151 / 2$ & 23 & 33 & $451 / 2$ & $661 / 2$ & - & - & \begin{tabular}{|l} 
Minn- \\
ten
\end{tabular} \\
\hline Das Filtrat war: & trübe & trübe & trübe & $\left|\begin{array}{l}\text { fast } \\
\text { klar }\end{array}\right|$ & fast & klar & klar & klar & klar & - & - & - \\
\hline $\begin{array}{l}\text { 2. Mit Eisen- } \\
\text { hydroxyd ge- } \\
\text { klärtes Wasser } \\
\text { a) Niederschlag } \\
\text { aufs Filter } \\
\text { gebracht, in: }\end{array}$ & $21 / 2$ & 5 & $81 / 4$ & - & 16 & $211 / 4$ & - & 32 & $381 / 4$ & 59 & - & Minu- \\
\hline Das Filtrat war: & klar & klar & klar & klar & klar & klar & klar & klar & klar & klar & - & - \\
\hline $\begin{array}{l}\text { b) Geklärtes } \\
\text { Wasser ohne } \\
\text { Niederschlag } \\
\text { aufs Filter } \\
\text { gebracht, in: }\end{array}$ & - & - & $31 / 4$ & - & - & $6 \%$ & - & - & $101 / 2$ & $141 / 2$ & $18 \%$ & $\int_{\text {ten }}^{M i n a-}$ \\
\hline
\end{tabular}

Hiernach wurde klares Wasser bei rohem Wasser erst nach $15 \frac{1}{2}$ Minuten erzielt, nachdem 5 Liter trübe abgelaufen waren. Die Filtrationsgeschwindigkeit nahm dann rasch $a b$, soda $B$ in den folgenden 51 Minuten nur 4 Liter klares Wasser erhalten wurden.

Bei dem mit dem Eisenoxydhydrat-Niederschlage auf das Filter gebrachten geklärten Wasser lief das Filtrat sofort klar $a b$, auch war die Verlangsamung der Filtration in den späteren Stadien des Versuches eine weit geringere wie bei dem rohen Wasser.

Das durch Absetzenlassen von dem Eisenhydroxyd-Niederschlage befreite Wasser zeigte, wie vorauszusehen, neben einer doppelt bis dreifach so großen Anfangsgeschwindigkeit später nur eine sehr unbedeutende Verlangsamung ${ }^{1}$ ). Das Filtrat war selbstverständlich auch hier von Anbeginn vollkommen klar.

Auch uber die Filtrationsgeschwindigkeit bei Beschickung des Filters mit einer großßeren Menge des Eisenhydroxyd-Niederschlages,

1) Die wohl auf die Quellung der Papierfasern zurückzuführen ist. 
also gewissermaßen einer künstlichen "Schleimschicht", habe ich Versuche angestellt, die zugleich auf die Bestimmung der organischen Substanz in den Filtraten mittelst Permanganat nach $\mathrm{Kubel}$ ausgedehnt warden.

$\mathrm{Zu}$ diesem Zwecke wurden $200 \mathrm{ccm}$ rohes Elbwasser mit $3 \mathrm{ccm}$ Fisenhydroxydlösung gemischt, unter öfterem Umrühren eine halbe Stunde lang stehen gelassen, und sodann das Gemisch nach gutem Umrühren aut das Filter gebracht und dieses bis zum Rande gefullt. Das Filtrat war vollkommen klar und farblos und trei von Eisenoxyd. Nachdem das Wasser abgelauten war, hatte sich der Niederschlag anscheinend ziemlich gleichmäßig auf dem Filter abgelagert, nach unten naturgemäß etwas stärker. Es wurde nun rohes Elbwasser nachgefullt und das Filter beständig bis zum Rande des aufgelagerten Eisenhydroxyd-Niederschlages rollgehalten. Das Filtrat war sofort vollkommen blank und klar. Es gebrauchte zum Darchlauten

$$
\begin{aligned}
& \text { das erste Liter } 20 \text { Minuten, } \\
& \text { das zweite Liter } 45 \text { Minuten, } \\
& \text { weitere } 0,8 \text { Liter } 75 \text { Minuten. }
\end{aligned}
$$

Wir sehen also auch hier bei Filtration von rohem Wasser eine ganz bedeutende Abnahme der Filtrationsgeschwindigkeit. Es filtrierten in 140 Minuten nur 2,8 Liter klares Wasser, während ohne den Eisenhydroxyd-Niederschlag auf das Filter gebrachtes geklärtes Wasser in $18^{8} / 4$ Minufen 15 Liter reines, klares Wasser lieferte.

Die Bestimmung der organischen Substanz ergab folgende Resultate:

Die zuerst mit dem Eisenhydroxyd-Niederschlage auf das Filter gebrachten $200 \mathrm{ccm}$ Wasser ergaben ein Filtrat, welches auf 100000 Teile 3,4 Teile organische Subitanz enthielt.

Im ersten Liter des nachfolgenden Filtrates yon dem mit rohem Elbwasser gespeisten Filter wurden gefunden

auf 100000 Teile 3,9 Teile organische Substanz, im zweiten Liter des nachfolgenden Filtrates 5,0 Teile, in den 0,8 Liter des dritten Filtrats 6,1 Teile, im Gesamtgemisch der Filtrate (3 Liter) 5,1 Teile organische Substanz auf 100000 Teile oder annăhernd dieselbe Menge, welche oben ${ }^{2}$ ) in dem im Verhältnis 1:1000 geklärten Elbwasser gefunden worden ist.

Die reinigende Wirkung, auf den Gehalt an organischer Substanz bezogen, ist hiernach ungefahr gleich groß, gleichviel ob man die gesamte Menge des zu reinigenden Wassers mit der entsprechenden Menge Eisenbydroxydlösung mischt und dann nach dem Absetzen ab-

1) Vergl. die Tabelle Seite 16. 
filtriert, oder ob man die der gesamten Menge des zu reinigenden Wassers entsprechende Menge der Eisenhydroxydlossang zuerst einer wesentlich kleineren Menge des rohen Wassers zuf ügt - bei obigem Versuch war es nur $1 / 15$ der Gesamtmenge -, dann den Niederschlag auf das Filter bringt, und die übrige der angewandten Menge Eisenhydroxydlösung entsprechende Menge Rohwasser nachfüllt.

Die bei letzterem Verfabren eintretende Verringerung der Filtrationsgeschwindigkeit ist aber so bedeutend, daB dem ersteren Verfahren unbedingt der Vorzug zu geben ist, besonders da das Absetzen in der Regel schon nach $1 / 2$ bis 1 Stunde ziemlich vollständig eintritt.

Ein bedentender Vorteil dürfte dabei für den Großbetrieb dadurch erwachsen, daß die Filter weit einfacher konstruiert werden kønnen, wenn sie nur den Zweck erfüllen, die geringen Reste des übrigens ziemlich großflockigen Niederschlages von Eisenhydroxyd zurtickzuhalten, welche etwa noch mit auf das Filter gelangen. Infolge der bedeutend größeren Filtrationsgeschwindigkeit kann die Ausdehnung der Filteranlagen eine weit beschränktere sein, um dabei doch dasselbe Quantum reinen Wassers zu liefern. Besonders aber wtirde bei der Reinigung des Wassers mittelst Eisenhydroxyd der oben (S. 18) erwähnte, von Fränkel und Piefke testgestellte, große Uebelstand vermieden werden, daß Anfang und Ende jeder Gebrauchsperiode der Sandfilter besonders gefährliche Zeiten sind, und $\mathrm{da} B$ das Ablanfenlassen des Filtrates und damit eine nicht unbedeutende Wasservergeudung nach jeder sich ziemlich oft wiederholenden Reinigung der Filter solange nötig wird, bis sich die "Schleimschicht" wieder in genügender Dicke gebildet hat. Auch würden die Filter eine stetige sehr lange Wirkungsdauer erhalten.

Der Anwendung des Verfahrens im Großbetriebe stand bisher die Umständlichkeit und Langwierigkeit der Herstellung der kolloidalen Eisenhydroxydlősung, welche nur durch Dialyse zu erzielen war, hindernd im Wege.

Man hat deshalb auch wohl mehrfach die einfacher und leichter herzustellende Eisenoxychloridflüssigkeit (den Liquor Ferri oxychlorati des Deutschen Arzneibuches) zu dem Zwecke empfohlen, welcher ja auch eine nicht geringe Menge kolloidalen Eisenhydroxyds enthält. Dieser aber erweist sich doch weit weniger dazu geeignet, wie die kolloidale Eisenhydroxydlösung (der Liquor Ferri oxydati dialysatus).

Zunăchst enthält der Liq. Ferri oxychlorati einen bedeutend höheren Chlorgehalt; infolgedessen ist er weniger leicht vollständig koaguliebar, and es erwächst die Gefahr, daß durch einen tibermäßigen Zusatz das Wasser eisenhaltig wird, und daß die gefallten organischen Eisenoxydverbindungen durch einen Ueberschuß des Fällungsmittels 
wieder in Lösung gehen. Ferner führt Oxychloridflussigkeit intolge ihres wesentlich höheren Chlorgehaltes dem Wasser mehr Chlorverbindangen zu, als die kolloidale Eisenhydroxydlosung.

Es ist deshalb mein Bestreben gewesen, ein einfaches und billiges Verfahren ausfindig za machen, um eine alle Eigenschaften des kolloidalen Eisenhydroxyds zeigende Lösung herzustellen, obne die amstăndliche und langwierige Dialyse.

Dies ist mir nun nach jahrelangen Versuchen und Untersuchungen, wie ich glanbe, in zufriedenstellender Weise endlich gelungen. Das diesbeztigliche Verfahren habe ich zum Patent angemeldet. Das Kaiserl. Patentamt hat dann auch die Erteilnng des Patentes am 31. Mai 1906 unter No. 173773 beschlossen, mit der Maßgabe, daß das Patent vom 8. Juli 1904 an läuft.

Ueber die Herstellungsart der Eisenhydroxydlösung enthălt die nachfolgend abgedruckte Patentschrift das Nähere.

\section{Beschreibang.}

Die vorliegende Erfindung betrifft die Herstellung einer Losung von Eisenhydroxyd, welche die Eigenschaften der kolloidalen Eisenhydroxydlosung besitzt und sich wegen ihrer billigen und einfachen Herstellungsweise zur Reinigung von Wasser im Großbetriebe eignet.

Die Darstellang dieser Lösung geschieht wie folgt:

Zu einer mäßig verdünnten Eisenchloridlosung, welche frei von Schwefelsăure ist, wird in kleineren Portionen eine Lösung von Natriumkarbonat oder Natriumbikarbonat, die gleichfalls møglichst frei von Schwefelsäure sein muB, in der Weise hinzugefügt, daB man den entstehenden Niederschlag sich immer erst wieder auflösen läBt, bevor man eine neue Menge der Natriumkarbonatlosung hinzufügt. Dieses wird so lange fortgesetzt, bis eine Probe der Fltissigkeit, mit Wasser verdünnt, auf Zusatz von Rhodansalzen nicht mehr oder doch nur ganz schwach blutrot gefärbt wird, aber noch eine im durchfallenden Lichte klare Losung darstellt.

Ist dieses erreicht, so fügt man vorsichtig noch soviel von einer stark verdünnten Lösung von Natriumkarbonat oder von Natriumbikarbonat hinzu, daß sich das Eisenhydroxyd abscheidet, die Flüssigkeit nach dem Absetzen oder Abfiltrieren farblos erscheint, aber noch schwach sauer bleibt oder neutral nicht aber alkalisch wird.

Den entstandenen Niederschlag sammelt man nach dem Absetzen, labt ihn vollkommen abtropfen und wäscht ihn durch Aufgießen von kleineren Mengen Wasser so lange aus, bis sich entwedor das abtropfende Wasser stärker gelb zu färben anfängt, oder aber nur noch schwache Chlorreaktion zeigt. 
Sodann befreit man den Niederschlag durch völliges Abtropfen and Absaugen auf einer Wasser gut aufsangenden Unterlage ${ }^{1}$ ) soweit von Feuchtigkeit, daß er in seiner Masse Risse bildet und sich leicht von der Unterlage abheben läßt, uad löst ihn dann durch Anrühren mit Wasser auf.

Wenn bei dez Fällung des Eisenhydroxyds die Flüssigkeit noch schwach sauer blieb, und beim Auswaschen sich das abtropfende Wasser gegen Ende gelb zu tärben anfing, so wird sich der Niederschlag jetzt leicht und klar im Wasser auflosen, und die Lösung wird nur im auffallenden, nicht aber im durchfallenden Lichte wenig trübe erscheinen. War hingegen die Flüssigkeit nach der Fällnng neutral, so tritt keive klare Lösung ein.

Im letzteren Falle, wenn keine klare Lösung eintritt, fügt man noch soviel Eisenchlorid hinzu, daß das Verhaltnis des Eisengehaltes (als Metall berechnet) zum Chlorgehalte in der Lösung nicht mehr betrăgt als anf je 3,5 Teile Eisen $0,6-0,7$ Teile Chlor, oder soviel Eisenchlorid, daß beim Erwärmen soeben klare Lösung erzielt wird, die Lorsung jedoch nicht mit Rhodansalzen reagiert.

Die hierzu nötige Menge Eisenchlorid kann man zweckmäßig auch in einer aliquoten Menge des Niederschlages durch einen vorsichtig angestellten Probeversuch feststellen.

Die erhaltene Lösung soll eine vollkommen klare dunkelbraunrote Flüssigkeit von schwach saurer Reaktion darstellen, welche auf das spez. Gew. 1,050-1,051 gebracht in 100 Teilen annähernd 3,5 Teile Eisen enthalt. Mit Wasser zwanzigfach verdünnt und zum Sieden erhitzt, soll sie sich nicht trüben, und sich weder mit Rhodansalzen blutrot farben, noch aus Kaliumjodid Jod frei machen. Sie wird sowohl durch Alkalien wie auch durch Säuren und durch viele Neutralsalze koaguliert, zeigt also alle chemischen Eigenschaften der kolloidalen Fisenhydroxydlösung, bezw. des Liquor Ferri oxydati dialysati. Durch ihr Verhalten gegen Rhodansalze und Jodkalium unterscheidet sich die nach dem vorliegenden Verfahren darzustellende Eisenlðsung scharf von einer Eisenoxychloridlösung, wie sie nach der Vorschrift des Deutschen Arzneibuches und nach Hager, pharmazeutisches-technisches Manuale 7. Aufl,, 1903, S. 422 durch Auflösen von frisch gefalltem Eisenhydroxyd in wenig Salzsăure erhalten wird.

Die Vergleichung einer nach dem vorliegenden Verfahren hergestellten Lðsung von Eisenhydroxyd mit einem aus der chemischen Fabrik von E. Merck in Darmstadt bezogenen nach der Vorschrift des Ergkinzungsbuches zum Deutschen Arzneibuche ${ }^{2}$ ) durch Dialyse

1) Oter darch Zentrifugieren.

s) Heransgegeben vom Deutschen Apotheker-Verein. 
aus Iiquor Ferri oxychlorati hergestellten Liquor Ferri oxydati dialysati ergab ein völlig tbereinstimmendes Verhalten beider Präparate gegen Reagentien.

\begin{tabular}{|c|c|c|c|c|c|c|c|c|}
\hline Präparat & 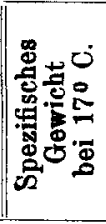 & 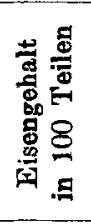 & 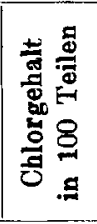 & 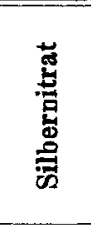 & 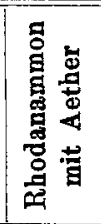 & 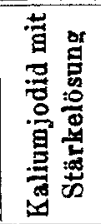 & 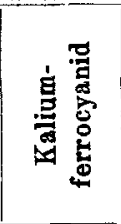 & 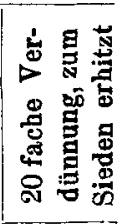 \\
\hline $\begin{array}{l}\text { Liq. Ferri } \\
\text { oxydati } \\
\text { dialysati } \\
\text { (Merck) }\end{array}$ & 1,04996 & 3,680 & 0,3918 & $\begin{array}{l}\text { keine } \\
\text { Trü- } \\
\text { bung }\end{array}$ & $\begin{array}{c}\text { blieb } \\
\text { farblos }\end{array}$ & $\begin{array}{c}\text { keine } \\
\text { dankle } \\
\text { Fär- } \\
\text { bung }\end{array}$ & $\begin{array}{l}\text { keine } \\
\text { Bildung } \\
\text { von } \\
\text { Berliner } \\
\text { Blau }\end{array}$ & $\begin{array}{c}\text { blieb } \\
\text { klar und } \\
\text { unver- } \\
\text { ändert }\end{array}$ \\
\hline $\begin{array}{c}\text { Eisen- } \\
\text { hydroxyd- } \\
\text { lösung } \\
\text { (Schweikert) }\end{array}$ & 1,04953 & 3,548 & 0,4730 & $\begin{array}{l}\text { keine } \\
\text { Trü- } \\
\text { bung }\end{array}$ & $\begin{array}{l}\text { blieb } \\
\text { farblos }\end{array}$ & $\begin{array}{c}\text { keine } \\
\text { dunkle } \\
\text { Fär- } \\
\text { bung }\end{array}$ & $\begin{array}{l}\text { keine } \\
\text { Bildung } \\
\text { von } \\
\text { Berliner } \\
\text { Blau }\end{array}$ & $\begin{array}{c}\text { blieb } \\
\text { klar und } \\
\text { unver- } \\
\text { ändert }\end{array}$ \\
\hline
\end{tabular}

Die Kosten der Herstellung der Eisenhydroxydlösung nach dem vorliegenden Verfahren, die für die Verwendung derselben im Großbetriebe naturgemä $\beta$ wesentlich ausschlaggebend sind, sind so gering, daß sie kein Hindernis für seine Einführung bilden dürften. Zudem bedarf es keiner besonderen komplizierten Apparate und Einrichtungen für die Gewinnung der betreffenden Flüssigkeit, welche auf den Wasserwerken selbst leicht vorgenommen werden kann.

Nach vorläufigen Ermittelungen und Berechnangen stellt sich der Preis der Rohmaterialien, welche zur Herstellung von 1000 Liter einer Eisenhydroxydlösung mit $3,5 \%$ Eisengehalt und einem spez. Gewicht von 1,050 nottig sind, etwa wie folgt:

$38 \mathrm{~kg}$ Eisenspäne $(\% \mathrm{~kg}=4,-\mathrm{M})$. . . . . . . . 1,52 M

290 n Salzsäure $(33 \% \mathrm{ig})$ arsenfrei $(\% \mathrm{~kg}=4,75 \mathrm{M})$. 13,78 ,

34 ” Braunstein ( $85 \% \mathrm{ig})(\% \mathrm{~kg}=15,-\mathrm{M}) . . \quad .5,10$ ”

116 " kalzinierte Soda $(90 \% \mathrm{ig})(\% \mathrm{~kg}=13,50 \mathrm{M})$ : . 11,30 .

Summa $31,70 \mathrm{M}$

Da mit 1000 Liter Eisenhydroxydlơsung im allgemeinen $1000 \mathrm{cbm}$ Wasser gereinigt werden können, so würden sich demnach die Kosten der Rohmaterialien für die Reinigung von ein Kubikmeter Wasser auf 3,2 Pfennige stellen.

Bei der Darstellung der Eisenhydroxydlösung entstehen aber noch Nebenprodukte, nämlich Wasserstoffgas, Manganchlorür, Chlornatrium und Kohlensäure, ferner fällt bei der Reinigung des Wassers 
Eisenhydroxyd ab. Inwieweit sich diese anderweit vorteilhaft nutz. bar machen lassen, ist eine besondere Frage, die ich hier nar streifen will:

Beim Auflösen des Eisens in Salzsăure erbălt man ans der obigen Menge von $36,75 \mathrm{~kg}$ Eisen 1,3 kg Wasserstoffgas oder annähernd $15 \mathrm{cbm}$, die vielleicht als Beimischung zum Leuchtgase zur Erhöhung seiner Heizkraft verwendet werden könnten. Auch das bei der Chloridierung des Eisenchlorürs zu Eisenchlorid entstehende Manganchlorür ist jedenfalls von gewissem Werte. Fraglicher erscheint, ob sich das bei der Klärung des Wassers niederfallende und mit Schmutzstoffen aus dem Wasser beladene noch chlorhaltige Eisenbydroxyd, welches sich bei gröBeren Wasserwerken- in bedeutenden Mengen ansammeln würde, nutzbar verwerten ließe. Aber es will mir nicht unmöglich erscheinen, daß dasgelbe sich wieder verarbeiten ließe, vielleicht auch nach Reinigung von den Schmutzteilen wieder im Kreislauf des Prozesses in Eisenchlorid umgewandelt werden könnte.

Gelänge die Einführung solcher Nebenprozesse, so wăren dadurch die Kosten des Verfahrens auf ein Minimum zurückgeführt.

Aber auch im anderen Falle erscheint bei dem geringen Preise der Rohmaterialien, der Einfachheit der Herstellung und Anwendung der Eisenhydroxydlösung und ihrer unzweifelhaft großen Brauchbarkeit für die Wasserreinigung eine Prüfung des Verfahrens im Großbetriebe mit Rücksicht auf die oben (S. 21 Abs. 2) erwhbnten großen Vorteile sicher gerechtfertigt, zumal wo es sich um Leben und Gesundheit vieler Merschen handelt.

Mitteilung aus dem chemischen Untersuchungsamte der Stadt Aachen.

\section{Eine neue Quecksilberlösung als Reagens auf Aldehyde, insbesondere Formaldehyd.}

Von E. Feder.

(Eingegangen den 25. XI. 1906.)

Eine allgemeine Reaktion auf Aldehyde ergibt sich bekanntlich aus dem Verhalten derselben zu NeBler's Reagens; sie geben mit letzterem einen zunächst rotbraunen Niederschlag, der sich alsbald grau färbt. Weiter sind auch die Sachse'sche und die Knapp'sche Quecksilberlösang, die beide als Reagens aut Traubenzucker im Urin empfohlen sind, zur Untersuchung aut Aldehyde zu verwerten. Die Sachse'sche 